\title{
Extremal $\boldsymbol{H}$-free planar graphs
}

\author{
Yongxin Lan* Yongtang Shi* \\ Center for Combinatorics and LPMC \\ Nankai University \\ Tianjin, China \\ yxlan0@126.com \\ shi@nankai.edu.cn \\ Zi-Xia Song ${ }^{\dagger}$ \\ Department of Mathematics \\ University of Central Florida \\ Orlando, U.S.A. \\ Zixia.Song@ucf .edu
}

Submitted: Oct 21, 2018; Accepted: Mar 28, 2019; Published: May 3, 2019

(C) The authors. Released under the CC BY-ND license (International 4.0).

\begin{abstract}
Given a graph $H$, a graph is $H$-free if it does not contain $H$ as a subgraph. We continue to study the topic of "extremal" planar graphs initiated by Dowden [J. Graph Theory 83 (2016) 213-230], that is, how many edges can an $H$-free planar graph on $n$ vertices have? We define $e x_{\mathcal{P}}(n, H)$ to be the maximum number of edges in an $H$-free planar graph on $n$ vertices. We first obtain several sufficient conditions on $H$ which yield $e x_{\mathcal{P}}(n, H)=3 n-6$ for all $n \geqslant|V(H)|$. We discover that the chromatic number of $H$ does not play a role, as in the celebrated ErdösStone Theorem. We then completely determine $e x_{\mathcal{P}}(n, H)$ when $H$ is a wheel or a star. Finally, we examine the case when $H$ is a $(t, r)$-fan, that is, $H$ is isomorphic to $K_{1}+t K_{r-1}$, where $t \geqslant 2$ and $r \geqslant 3$ are integers. However, determining $e x_{\mathcal{p}}(n, H)$, when $H$ is a planar subcubic graph, remains wide open.
\end{abstract}

Mathematics Subject Classifications: 05C10, 05C35

* Supported by National Natural Science Foundation of China, Natural Science Foundation of Tianjin (No. 17JCQNJC00300), the China-Slovenia bilateral project "Some topics in modern graph theory" (No. 12-6), and the Fundamental Research Funds for the Central Universities, Nankai University (63191516).

†Corresponding author. 


\section{Introduction}

All graphs considered in this paper are finite and simple. We use $K_{t}, C_{t}$ and $P_{t}$ to denote the complete graph, cycle, and path on $t$ vertices, respectively. Given a graph $G$, we will use $V(G)$ to denote the vertex set, $E(G)$ the edge set, $|G|$ the number of vertices, $e(G)$ the number of edges, $\delta(G)$ the minimum degree, $\Delta(G)$ the maximum degree. For a vertex $v \in V(G)$, we will use $N_{G}(v)$ to denote the set of vertices in $G$ which are adjacent to $v$. Let $d_{G}(v)=\left|N_{G}(v)\right|$ denote the degree of the vertex $v$ in $G$ and $N_{G}[v]=N_{G}(v) \cup\{v\}$. A vertex is a $k$-vertex in $G$ if it has degree $k$. We use $n_{k}(G)$ to denote the number of $k$-vertices in $G$. For any set $S \subset V(G)$, the subgraph of $G$ induced on $S$, denoted $G[S]$, is the graph with vertex set $S$ and edge set $\{x y \in E(G): x, y \in S\}$. We denote by $G \backslash S$ the subgraph of $G$ induced on $V(G) \backslash S$. If $S=\{v\}$, then we simple write $G \backslash v$. For any two disjoint sets $A$ and $B$ of $V(G)$, we use $e_{G}(A, B)$ to denote the number of edges between $A$ and $B$ in $G$. The join $G+H$ (resp. union $G \cup H$ ) of two vertex-disjoint graphs $G$ and $H$ is the graph having vertex set $V(G) \cup V(H)$ and edge set $E(G) \cup E(H) \cup\{x y \mid x \in V(G), y \in V(H)\}$ (resp. $E(G) \cup E(H)$ ). For a positive integer $t$, we use $t G$ to denote disjoint union of $t$ copies of a graph $G$. Given two isomorphic graphs $G$ and $H$, we may (with a slight but common abuse of notation) write $G=H$. For any positive integer $k$, let $[k]:=\{1,2, \ldots, k\}$.

Given a graph $H$, a graph is $H$-free if it does not contain $H$ as a subgraph. One of the best known results in extremal graph theory is Turán's Theorem [15], which gives the maximum number of edges that a $K_{t}$-free graph on $n$ vertices can have. The celebrated Erdös-Stone Theorem [7] then extends this to the case when $K_{t}$ is replaced by an arbitrary graph $H$ with at least one edge, showing that the maximum number of edges possible is $(1+o(1))\left(\begin{array}{l}n \\ 2\end{array}\right)\left(\frac{\chi(H)-2}{\chi(H)-1}\right)$, where $\chi(H)$ denotes the chromatic number of $H$. This latter result has been called the "fundamental theorem of extremal graph theory" [1]. Turán-type problems when host graphs are hypergraphs are notoriously difficult. A large quantity of work in this area has been carried out in determining the maximum number of edges in a $k$-uniform hypergraph on $n$ vertices without containing $k$-uniform linear paths and cycles (see, for example, $[9,10,12])$. Surveys on Turán-type problems of graphs and hypergraphs can be found in $[8,11]$.

In this paper, we continue to study the topic of "extremal" planar graphs, that is, how many edges can an $H$-free planar graph on $n$ vertices have? We define $e x_{\mathcal{p}}(n, H)$ to be the maximum number of edges in an $H$-free planar graph on $n$ vertices. Dowden [3] initiated the study of $e x_{\mathcal{p}}(n, H)$ and proved the following results, where each bound is tight.

Theorem 1 ([3]). Let $n$ be a positive integer.

(a) $e x_{\mathcal{p}}\left(n, C_{4}\right) \leqslant 15(n-2) / 7$ for all $n \geqslant 4$.

(b) $e x_{\mathcal{p}}\left(n, C_{5}\right) \leqslant(12 n-33) / 5$ for all $n \geqslant 11$.

Let $\Theta_{4}$ and $\Theta_{5}$ be obtained from $C_{4}$ and $C_{5}$, respectively, by adding an additional edge joining two non-consecutive vertices. The present authors [13] studied $e x_{\mathcal{P}}(n, H)$ when 
$H \in\left\{\Theta_{4}, \Theta_{5}, C_{6}\right\}$ and when $H$ is a path on at most 9 vertices. Results from [13] are summarized below.

Theorem 2 ([13]). Let $n$ be a positive integer. Then

(a) $e x_{\mathcal{P}}\left(n, \Theta_{4}\right) \leqslant 12(n-2) / 5$ for all $n \geqslant 4$, with equality when $n \equiv 12(\bmod 20)$.

(b) $e x_{\mathcal{p}}\left(n, \Theta_{5}\right) \leqslant 5(n-2) / 2$ for all $n \geqslant 5$, with equality when $n \equiv 50(\bmod 120)$.

(c) $e x_{\mathcal{p}}\left(n, C_{6}\right) \leqslant 18(n-2) / 7$ for all $n \geqslant 6$.

(d) $e x_{\mathcal{P}}\left(n, P_{9}\right) \leqslant \max \{9 n / 4,(5 n-8) / 2\}$.

It seems quite non-trivial to determine $e x_{\mathcal{p}}\left(n, C_{t}\right)$ for all $t \geqslant 7$. In this paper, we first investigate planar graphs $H$ satisfying $e x_{\mathcal{p}}(n, H)=3 n-6$ for all $n \geqslant|H|$. This partially answers a question of Dowden [3]. As observed in [3], for all $n \geqslant 6$, the planar triangulation $2 K_{1}+C_{n-2}$ is $K_{4}$-free. Hence, $e x_{\mathcal{p}}(n, H)=3 n-6$ for all graphs $H$ which contains $K_{4}$ as a subgraph and $n \geqslant \max \{|H|, 6\}$. In particular, ex $\left(n, K_{5}^{-}\right)=3 n-6$ for all $n \geqslant 6$, where $K_{p}^{-}$denotes the graph obtained from $K_{p}$ by deleting one edge. Proposition 3 below describes several sufficient conditions on $K_{4}$-free planar graphs $H$ such that $e x_{\mathcal{P}}(n, H)=3 n-6$ for all $n \geqslant|H|$.

Proposition 3. Let $H$ be a $K_{4}$-free planar graph and let $n \geqslant|H|$ be an integer. Then $e x_{\mathcal{P}}(n, H)=3 n-6$ if one of the following holds.

(a) $\chi(H)=4$ and $n \geqslant|H|+2$.

(b) $\Delta(H) \geqslant 7$.

(c) $\Delta(H)=6$ and either $n_{6}(H)+n_{5}(H) \geqslant 2$ or $n_{6}(H)+n_{5}(H)=1$ and $n_{4}(H) \geqslant 5$.

(d) $\Delta(H)=5$ and either $H$ has at least three 5 -vertices or $H$ has exactly two adjacent 5-vertices.

(e) $\Delta(H)=4$ and $n_{4}(H) \geqslant 7$.

(f) $H$ is 3-regular with $|H| \geqslant 9$ or $H$ has at least three vertex-disjoint cycles or $H$ has exactly one vertex $u$ of degree $\Delta(H) \in\{4,5,6\}$ such that $\Delta(H[N(u)]) \geqslant 3$.

(g) $\delta(H) \geqslant 4$ or $H$ has exactly one vertex of degree at most 3 .

Proposition 3 implies that $e x_{\mathcal{p}}(n, H)=3 n-6$ for all $H$ with $n \geqslant|H|+2$ and either $\chi(H)=4$ or $\chi(H)=3$ and $\Delta(H) \geqslant 7$. Note that $\Theta_{4}=K_{4}^{-}$, and both $K_{4}^{-}$and $K_{1}+2 K_{2}$ have chromatic number 3. Theorem 2(a) and Theorem 5 (see below) then demonstrate that the chromatic number of $H$ does not play a role, as in the Erdös-Stone Theorem.

By Proposition $3, e x_{\mathcal{p}}(n, H)$ remains unknown for $K_{4}$-free planar graphs $H$ with $\Delta(H)=6, n_{6}(H)+n_{5}(H)=1$ and $n_{4}(H) \leqslant 4$; or $\Delta(H)=5$ and $n_{5}(H) \leqslant 2$ (and the two 5 -vertices are not adjacent when $n_{5}(H)=2$ ); or $\Delta(H)=4$ and $n_{4}(H) \leqslant 6$; or $\Delta(H) \leqslant 3$. In particular, by Proposition $3(f), e x_{\mathcal{p}}(n, H)$ remains unknown for $K_{4}$-free planar graphs $H$ with exactly one vertex, say $u$, of degree $\Delta(H) \leqslant 6$ and $\Delta(H[N(u)]) \leqslant 2$. It seems non-trivial to determine $e x_{\mathcal{p}}(n, H)$ for all such $H$. We next study $\operatorname{ex}_{\mathcal{p}}\left(n, W_{k}\right)$, where $W_{k}:=K_{1}+C_{k}$ is a wheel on $k+1 \geqslant 5$ vertices. Unlike the classic Turán number of $W_{k}$ (see $[4,5]$ for more information), the planar Turán number of $W_{k}$ can be completely determined. We establish this in Theorem 4. 
Theorem 4. Let $n, k$ be integers with $n \geqslant k+1 \geqslant 5$. Then

$$
e x_{\mathcal{P}}\left(n, W_{k}\right)= \begin{cases}3 n-6 & \text { if } k \geqslant 6, \text { or } k=5 \text { and } n \neq 7, \text { or } k=4 \text { and } n \geqslant 12 \\ 3 n-7 & \text { if } k=4 \text { and } n \in\{5,6\}, \text { or } k=5 \text { and } n=7 \\ 3 n-8 & \text { if } k=4 \text { and } 7 \leqslant n \leqslant 11 .\end{cases}
$$

A graph is a $(t, r)$-fan if it is isomorphic to $K_{1}+t K_{r-1}$, where $t \geqslant 2$ and $r \geqslant 2$ are integers. The classical Turán number of $(t, r)$-fan, namely, ex $\left(n, K_{1}+t K_{r-1}\right)$, has also been studied when $n$ is sufficiently large (see [6,2] for more information). We next study $e x_{\mathcal{P}}(n, H)$ when $H$ is a $K_{4}$-free $(t, r)$-fan, in particular, when $H \in\left\{K_{1}+2 K_{2}, K_{1, t}, K_{1}+\right.$ $\left.3 K_{2}\right\}$. Theorem 5 below establishes a sharp upper bound for $e x_{\mathcal{p}}\left(n, K_{1}+2 K_{2}\right)$, and Theorem 6 completely determines the value of $e x_{\mathcal{p}}\left(n, K_{1, t}\right)$ for all $t \geqslant 3$. However, the upper bound for $e x_{\mathcal{p}}\left(n, K_{1}+3 K_{2}\right)$ when $n \geqslant 15$ in Theorem 7 is not tight.

Theorem 5. Let $n \geqslant 5$ be an integer. Then

$$
2 n-3 \leqslant e x_{\mathcal{P}}\left(n, K_{1}+2 K_{2}\right) \leqslant \frac{19 n}{8}-4 .
$$

Furthermore, $e x_{\mathcal{P}}\left(n, K_{1}+2 K_{2}\right)=\frac{19 n}{8}-4$ if and only if $n$ is divisible by 8 .

Theorem 6. Let $n, t$ be integers with $n \geqslant t+1 \geqslant 4$. Then

$$
e x_{\mathcal{P}}\left(n, K_{1, t}\right)= \begin{cases}3 n-6 & \text { if } t \geqslant 7, \text { or } t=6 \text { and } n \in\{7,8,9,10,12\} \\ 3 n-7 & \text { if } t=6 \text { and } n=11 \\ 3 n-8 & \text { if } t=6 \text { and } n \in\{13,14\}, \text { or } t=5 \text { and } n=7 \\ \left\lfloor\frac{(t-1) n}{2}\right\rfloor & \text { if } t \in\{3,4\}, \text { or } t=5 \text { and } n \neq 7, \text { or } t=6 \text { and } n \geqslant 15 .\end{cases}
$$

Theorem 7. Let $n \geqslant 7$ be an integer. Then

$$
\left\lfloor\frac{5 n}{2}\right\rfloor \leqslant e x_{\mathcal{P}}\left(n, K_{1}+3 K_{2}\right)<\frac{17 n}{6}-4
$$

for all $n \geqslant 15$ and

$$
e x_{\mathcal{P}}\left(n, K_{1}+3 K_{2}\right)= \begin{cases}3 n-6 & \text { if } n \in\{7,8,9,10,12\} \\ 3 n-7 & \text { if } n=11 \\ 3 n-8 & \text { if } n \in\{13,14\} .\end{cases}
$$

We need to introduce more notation. Given a plane graph $G$ and an integer $i \geqslant 3$, an $i$-face in $G$ is a face of order $i$. Let $f_{i}$ denote the number of $i$-faces in $G$. Let $T_{n}$ denote 
a plane triangulation on $n \geqslant 3$ vertices, and let $T_{n}^{-}$be obtained from $T_{n}$ with one edge removed. For every integer $n \geqslant 5$, let $O_{n}$ denote the unique outerplane graph with $2 n-3$ edges, maximum degree 4 , and the outer face of order $n$; let $O_{n}^{\prime}$ be a different drawing of $O_{n}$ with one unique inner face of order $n$; and finally, let $O_{n}^{*}$ be the planar triangulation obtained from $O_{n}$ and $O_{n}^{\prime}$ by identifying the outer face of $O_{n}$ with the unique $n$-face of $O_{n}^{\prime}$ in such a way that $O_{n}^{*}$ is a simple graph. The proof of Proposition 3 relies heavily on the fact that $O_{n}^{*}, K_{1}+O_{n-1}$ and $2 K_{1}+C_{n-2}$ are planar triangulations.

Finally, we shall make use of the following lemma in the proof of Theorem 6 and Theorem 7 .

Lemma 8 ([14]). There does not exist a 4-regular planar graph on 7 vertices, or a 5-regular planar graph on 14 vertices, or a planar graph on $n \in\{11,13\}$ vertices with exactly one vertex of degree 4 and $n-1$ vertices of degree 5 .

\section{Proof of Proposition 3}

Let $H$ and $n$ be given as in the statement. To prove $(a)$, assume $\chi(H)=4$ and $n \geqslant|H|+2$. Since the planar triangulation $2 K_{1}+C_{n-2}$ has no subgraph on $|H|$ vertices with chromatic number 4 , we see that $2 K_{1}+C_{n-2}$ is $H$-free. Hence, $e x_{\mathcal{P}}(n, H)=3 n-6$ when $\chi(H)=4$ and $n \geqslant|H|+2$.

To prove $(b)$, assume that $\Delta(H) \geqslant 7$. Then $n \geqslant|H| \geqslant 8$, and the planar triangulation $O_{n}^{*}$ is $H$-free because $\Delta\left(O_{n}^{*}\right)=6$. Hence, $e x_{\mathcal{p}}(n, H)=3 n-6$ for all $n \geqslant|H|$.

To prove $(c)$, assume $\Delta(H)=6$. Then $n \geqslant|H| \geqslant 7$. Assume first $n_{6}(H)+n_{5}(H) \geqslant 2$. Let $x, y \in V(H)$ be such that $d_{H}(x)=6$ and $d_{H}(y) \geqslant 5$. Then the planar triangulation $K_{1}+O_{n-1}$ is $H$-free when $x y \notin E(H)$, and the planar triangulation $2 K_{1}+C_{n-2}$ is $H$ free when $x y \in E(H)$. Hence, $e x_{\mathcal{p}}(n, H)=3 n-6$ when $n_{6}(H)+n_{5}(H) \geqslant 2$. Next assume that $n_{6}(H)+n_{5}(H)=1$ and $n_{4}(H) \geqslant 5$. Then $n_{5}(H)=0$. Note that the planar triangulation $2 K_{1}+C_{n-2}$ is $H$-free when $n_{4}(H) \geqslant 6$. We may further assume that $n_{4}(H)=5$. Let $u, v_{1}, \ldots, v_{5} \in V(H)$ with $d_{H}(u)=6$ and $d_{H}\left(v_{i}\right)=4$ for all $i \in[5]$. Let $M:=H\left[\left\{u, v_{1}, \ldots, v_{5}\right\}\right]$. We may assume that $u v_{1} \notin E(M)$ when $d_{M}(u)=4$. Then the planar triangulation $2 K_{1}+C_{n-2}$ is $H$-free when $d_{M}(u)=5$, or $d_{M}(u) \leqslant 3$, or $d_{M}(u)=4$ and $d_{M}\left(v_{1}\right) \leqslant 3$; and the planar triangulation $O_{n}^{*}$ is $H$-free when $N_{M}(u)=N_{M}\left(v_{1}\right)=$ $\left\{v_{2}, v_{3}, v_{4}, v_{5}\right\}$. It follows that $e x_{\mathcal{p}}(n, H)=3 n-6$ for all $n \geqslant|H|$. This proves $(c)$.

To prove $(d)$, assume $\Delta(H)=5$ and $n_{5}(H) \geqslant 2$. Then $n \geqslant|H| \geqslant 6$. Let $u, v$ be two distinct 5-vertices in $H$. Then either $n_{5}(H) \geqslant 3$ or $n_{5}(H)=2$ with $u v \in E(H)$. Note that the planar triangulation $2 K_{1}+C_{n-2}$ has exactly two non-adjacent vertices of degree at least 5 when $n \geqslant 7$ and has maximum degree 4 when $n=6$. Hence, $2 K_{1}+C_{n-2}$ is $H$-free, and so $e x_{\mathcal{p}}(n, H)=3 n-6$ for all $n \geqslant|H|$. This proves $(d)$.

To prove $(e)$ and $(f)$. Assume $\Delta(H)=4$ and $n_{4}(H) \geqslant 7$, or $H$ is 3-regular with $|H| \geqslant 9$, or $H$ has at least three vertex-disjoint cycles, or $H$ has exactly one vertex $u$ of degree $\Delta(H) \in\{4,5,6\}$ such that $\Delta(H[N(u)]) \geqslant 3$. Then the planar triangulation $2 K_{1}+C_{n-2}$ is $H$-free. Hence, $e x_{\mathcal{P}}(n, H)=3 n-6$ for all $n \geqslant|H|$. 
It remains to prove $(g)$. Assume $\delta(H) \geqslant 4$ or $H$ has exactly one vertex of degree at most 3. Then $n \geqslant|H| \geqslant 5$. Note that the planar triangulation $K_{1}+O_{n-1}$ is 3-degenerate and every subgraph of $K_{1}+O_{n-1}$ has at least two vertices of degree at most 3 , because every subgraph of $O_{n-1}$ has at least two vertices of degree at most 2 . Hence, $K_{1}+O_{n-1}$ is $H$-free, and so $e x_{\mathcal{P}}(n, H)=3 n-6$ for all $n \geqslant|H|$.

This completes the proof of Proposition 3.

\section{Proof of Theorem 4}

Let $n, k$ be given as in the statement. Assume $k \geqslant 7$. By Proposition $3(b), e x_{\mathcal{P}}\left(n, W_{k}\right)=$ $3 n-6$ for all $n \geqslant k+1$. Assume next $k \in\{5,6\}$. Since the planar triangulation $2 K_{1}+C_{n-2}$ is $W_{k}$-free when $n \geqslant k+3$ or $n=k+1$, we see that $e x_{\mathcal{p}}\left(n, W_{k}\right)=3 n-6$ when $n \geqslant k+3$ or $n=k+1$. We next determine $e x_{\mathcal{P}}\left(n, W_{k}\right)$ when $n=k+2$. For $k=6$ and $n=8$, the plane triangulation on 8 vertices depicted in Figure $1(\mathrm{a})$ is $W_{6}$-free and so $e x_{\mathcal{P}}\left(n, W_{6}\right)=3 n-6$ when $n=8$. For $k=5$ and $n=7$, note that the plane graph with 7 vertices and 14 edges

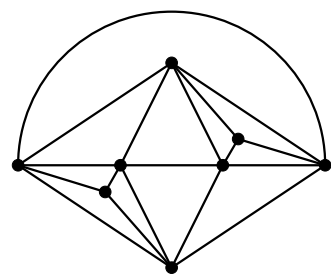

(a)

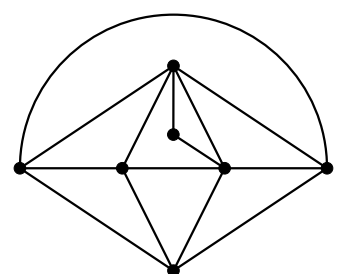

(b)

Figure 1: $W_{k}$-free plane graphs with $n=k+2$ vertices and $3 n-12+k$ edges, where $k \in\{5,6\}$.

given in Figure $1(\mathrm{~b})$ is $W_{5}$-free. Thus, $e x_{\mathcal{P}}\left(7, W_{5}\right) \geqslant 3 \cdot 7-6-1=14$. On the other hand, all plane triangulations on seven vertices ${ }^{1}$ are depicted in Figure 2, each containing a copy of $W_{5}$. Hence, ex $\left(n, W_{5}\right)=3 n-7$ when $n=7$.

It remains to consider the case when $k=4$. To show $e x_{\mathcal{p}}\left(n, W_{4}\right)=3 n-6$ for all $n \geqslant 12$, assume first that $n=5 t+2$ for some integer $t \geqslant 2$. Let $L_{t}$ be a plane triangulation on $n=5 t+2$ vertices constructed as follows: for each $i \in[t]$, let $C^{i}$ be a cycle with vertices $u_{i, 1}, u_{i, 2}, \ldots, u_{i, 5}$ in order. Let $L_{t}$ be the plane triangulation obtained from disjoint union of $C^{1}, \ldots, C^{t}$ by adding edges $u_{i, j} u_{i+1, j}$ and $u_{i, j} u_{i+1, j+1}$ for all $i \in[t-1]$ and $j \in[5]$, where

\footnotetext{
${ }^{1}$ To find all plane triangulations on 7 vertices, let $H$ be a plane triangulation on 7 vertices. Then $e(H)=15$ and $H$ must be 3 -connected with maximum degree 5 or 6 . Let $u \in V(H)$ be a vertex of maximum degree. If $d(u)=6$, then $H$ has at most two vertices of degree 6 and $H-u$ has neither $K_{4}$ nor $K_{2,3}$ minor. Thus $H-u$ must be outer planar and so $H$ is isomorphic to one of the plane triangulations in Figure $2(\mathrm{a}, \mathrm{b}, \mathrm{e})$. If $d(u)=5$, then the degree sequence of $H$ is either $(5,5,5,4,4,4,3)$ or $(5,5,4,4,4,4,4)$. Since $H$ has no $K_{3,3}$ minor, one can then check that $H$ is isomorphic to one of the plane triangulations in Figure 2(c,d).
} 


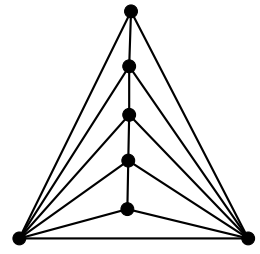

(a)

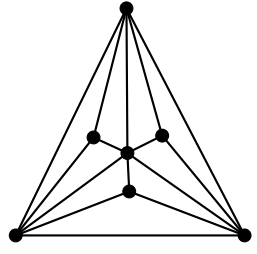

(b)

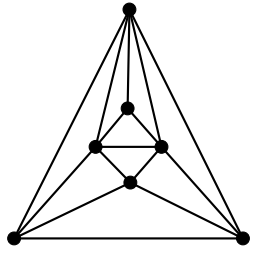

$(c)$

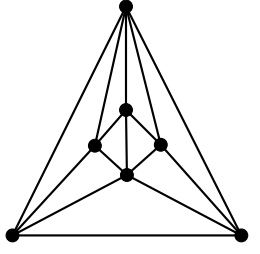

$(d)$

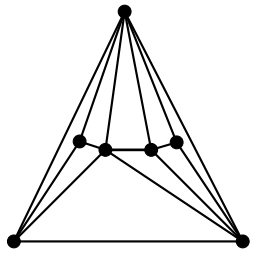

$(e)$

Figure 2: All plane triangulations on 7 vertices. Furthermore, each $T_{7}$ has a copy of $W_{4}$ and $W_{5}$, and each $T_{7}^{-}$has a copy of $W_{4}$.

all arithmetic on the index $j+1$ here is done modulo 5, and finally adding two new nonadjacent vertices $u$ and $v$ such that $u$ is adjacent to all vertices of $C^{1}$ and $v$ is adjacent to all vertices of $C^{t}$. The graph $L_{t}$ when $t=3$ is depicted in Figure 3 . It is worth noting that $L_{t}$ is $K_{4}$-free, $d_{L_{t}}(u)=d_{L_{t}}(v)=5, d_{L_{t}}\left(u_{1, j}\right)=d_{L_{t}}\left(u_{t, j}\right)=5, d_{L_{t}}\left(u_{i, j}\right)=6$ for $2 \leqslant i \leqslant t-1$ and $j \in[5]$. Furthermore, the subgraph induced by the neighborhood of each vertex in $L_{t}$ is isomorphic to either $C_{5}$ or $C_{6}$. Hence, $L_{t}$ is $W_{4}$-free and so $e x_{\mathcal{p}}\left(n, W_{4}\right)=3 n-6$ when $n=5 t+2$ for some integer $t \geqslant 2$.

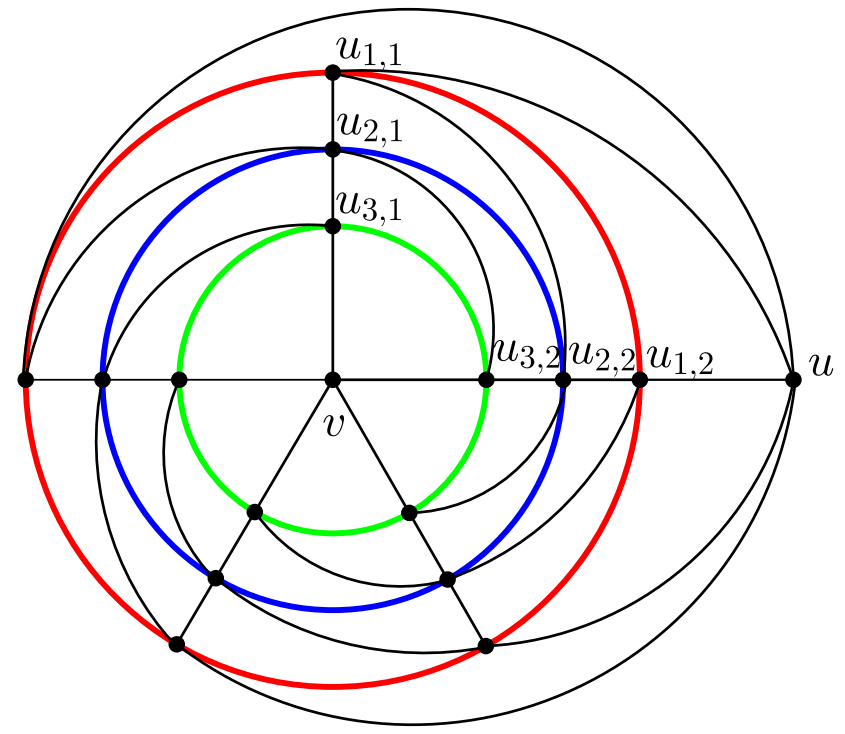

Figure 3: The plane triangulation $L_{t}$ when $t=3$, where $C^{1}, C^{2}$ and $C^{3}$ are in red, blue and green, respectively.

Next assume that $n=5 t+2+i$ for some $i \in[4]$, where $t \geqslant 2$ is an integer. Note that the plane triangulation $L_{t}$ on $5 t+2$ vertices constructed above contains at least four pairwise vertex-disjoint faces. Let $F_{1}, \ldots, F_{i}$ be any $i$ pairwise vertex-disjoint faces of $L_{t}$, and let $L_{t}^{i}$ be the plane triangulation obtained from $L_{t}$ by adding one vertex, say $x_{j}$, of degree 3 to each $F_{j}$ for all $j \in[i]$. Clearly, $L_{t}^{i}$ is a plane triangulation on $n=5 t+2+i$ 
vertices. By the choice of $F_{1}, \ldots, F_{i}$, we see that $x_{1}, \ldots, x_{i}$ are pairwise non-adjacent in $L_{t}^{i}$, and no two of $x_{1}, \ldots, x_{i}$ have common neighbors in $L_{t}^{i}$. We next show that $L_{t}^{i}$ is $W_{4}$-free for all $i \in[4]$. Suppose that $L_{t}^{i}$ contains a copy of $W_{4}$ for some $i \in$ [4]. Let $H$ be a $W_{4}$ in $L_{t}^{i}$. Then $H$ must contain exactly one, say $x_{1}$, of $x_{1}, \ldots, x_{i}$, because $L_{t}$ is $W_{4}$-free, and no two of $x_{1}, \ldots, x_{i}$ are adjacent or have common neighbors in $L_{t}^{i}$. Let $y, z \in V(H)$ be the two neighbors of $x_{1}$ such that $y z \notin E(H)$. By the choice of $x_{1}$, we see that $y z \in E\left(L_{t}\right)$. But then $L_{t}\left[V\left(H \backslash x_{1}\right)\right]=K_{4}$ and so $L_{t}$ contains $K_{4}$ as a subgraph, a contradiction. Therefore, $e x_{\mathcal{p}}\left(n, W_{4}\right)=3 n-6$ for all $n \geqslant 12$.

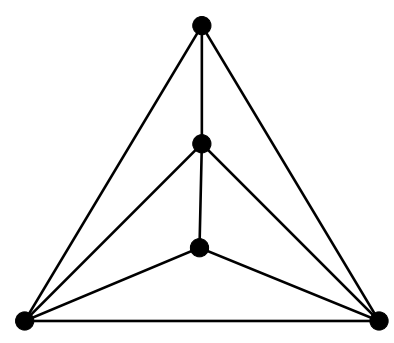

(a)

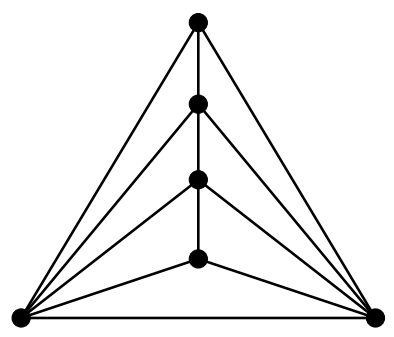

(b)

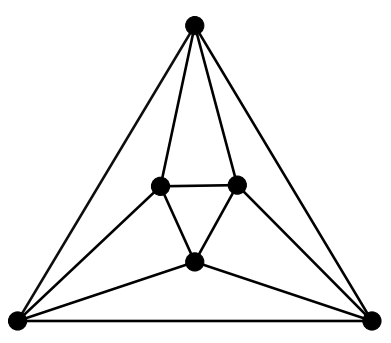

(c)

Figure 4: All plane triangulations on 5 and 6 vertices.

We next show that $e x_{\mathcal{p}}\left(n, W_{4}\right)=3 n-7$ when $n \in\{5,6\}$. Note that all plane triangulations on $n \in\{5,6\}$ vertices are depicted in Figure 4, each containing a copy of $W_{4}$. Thus, $e x_{\mathcal{P}}\left(n, W_{4}\right) \leqslant 3 n-7$. On the other hand, for all $n \in\{5,6\}$, the planar graph $K_{2}+\left(K_{2} \cup K_{n-4}\right)$ has $3 n-7$ edges and is $W_{4}$-free. Hence, $e x_{p}\left(n, W_{4}\right)=3 n-7$ when $n \in\{5,6\}$.

Finally, we show that $e x_{\mathcal{p}}\left(n, W_{4}\right)=3 n-8$ for all $n \in\{7,8,9,10,11\}$. The plane graph $J$, given in Figure 5 , is $W_{4}$-free with $n=11$ vertices and $3 n-8$ edges. Let $B$ be the set of

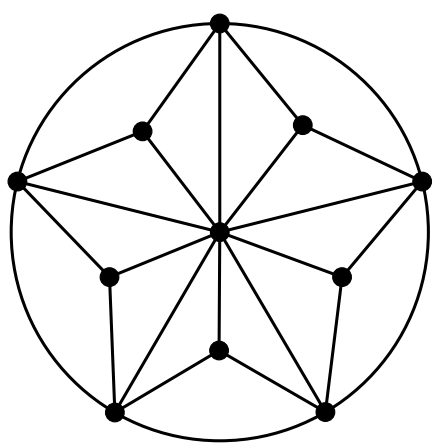

Figure 5: Graph $J$.

all vertices of degree 3 in $J$. Then $|B|=5$. For each $n \in\{7,8,9,10\}$, let $J_{n}$ be the plane graph obtained from $J$ by deleting $11-n$ vertices in $B$. Then $J_{n}$ is an induced subgraph of 
$J$. Clearly, $J_{n}$ is $W_{4}$-free with $n$ vertices and $3 n-8$ edges. Hence, ex $\left(n, W_{4}\right) \geqslant 3 n-8$ for all $n \in\{7,8,9,10,11\}$. We next show that $e x_{\mathcal{P}}\left(n, W_{4}\right) \leqslant 3 n-8$ for all $n \in\{7,8,9,10,11\}$. Suppose this is not true. Let $G$ be a $W_{4}$-free planar graph on $n \in\{7,8,9,10,11\}$ vertices with $e(G) \geqslant 3 n-7$. We choose such a $G$ with $n$ minimum. Then $G=T_{n}$ or $G=T_{n}^{-}$. Since each $T_{7}$, depicted in Figure 2, contains a copy of $W_{4}$, and each $T_{7}^{-}$also contains a copy of $W_{4}$, it follows that $n \in\{8,9,10,11\}$. Let $u \in V(G)$ with $d_{G}(u)=\delta(G)$. Then $\delta(G) \leqslant 4$, else $e(G) \geqslant \frac{5 n}{2}>3 n-6$ because $n \leqslant 11$, a contradiction. Next, if $\delta(G) \leqslant 3$, then $e(G \backslash u) \leqslant 3(n-1)-8$ by minimality of $n$ and the fact that $e x_{\mathcal{p}}\left(n, W_{4}\right) \leqslant 3 n-8$ when $n=7$. Thus, $e(G)=e(G \backslash u)+d_{G}(u) \leqslant 3(n-1)-8+3=3 n-8$, a contradiction. This proves that $\delta(G)=4$. Since $N_{G}[u]$ does not contain a copy of $W_{4}$ in $G$, we see that $G \neq T_{n}$. Thus $G=T_{n}^{-}$. We may assume that $G$ is a plane drawing of $T_{n}^{-}$such that the outer face is a 3-face. Let $x_{1}, y_{1} \in V(G)$ be such that $G+x_{1} y_{1}=T_{n}$. Then $x_{1}$ and $y_{1}$ must lie on the boundary of the unique 4 -face, say $F$, in $G$. Let $x_{1}, x_{2}, y_{1}, y_{2}$ be the vertices on the boundary of $F$ in order. Then $d_{G}(v) \geqslant 5$ for all $v \in V(G) \backslash\left\{x_{1}, x_{2}, y_{1}, y_{2}\right\}$, because $G=T_{n}^{-}$and $N_{G}[u]$ does not contain a copy of $W_{4}$ in $G$ for any $u \in V(G)$ with $d_{G}(u)=4$. Thus $2(3 n-7)=2 e(G) \geqslant 4 \cdot 4+5 \cdot(n-4)$, which implies that $n \in\{10,11\}$. Suppose each vertex in $\left\{x_{1}, x_{2}, y_{1}, y_{2}\right\}$ has degree 4 in $G$. Since $G=T_{n}^{-}$, there must exist four distinct vertices $z_{1}, z_{2}, z_{3}, z_{4} \in V(G) \backslash\left\{x_{1}, x_{2}, y_{1}, y_{2}\right\}$ such that $G[A]$ is isomorphic to the graph given in Figure $6(\mathrm{a})$, where $A=\left\{x_{1}, x_{2}, y_{1}, y_{2}, z_{1}, z_{2}, z_{3}, z_{4}\right\}$. But then $G$ contains $K_{3,3}$ as

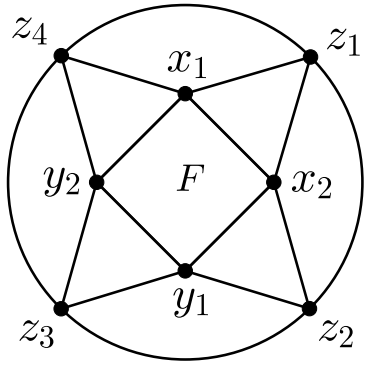

(a)

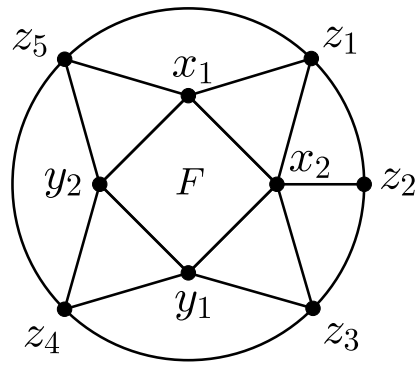

(b)

Figure 6: The graph $G[A]$.

a minor, because $n \in\{10,11\}$ and $d_{G}(v) \geqslant 5$ for any $v \in V(G) \backslash A$. Thus, we may assume that $d_{G}\left(x_{2}\right) \geqslant 5$. Then $2(3 n-7)=2 e(G) \geqslant 4 \cdot 3+5 \cdot(n-3)$, which implies that $n=11$, $d_{G}(v)=4$ for all $v \in\left\{x_{1}, y_{1}, y_{2}\right\}$ and $\Delta(G)=5$. Thus there exist five distinct vertices $z_{1}, z_{2}, z_{3}, z_{4}, z_{5} \in V(G) \backslash\left\{x_{1}, x_{2}, y_{1}, y_{2}\right\}$ such that $G[A]$ is isomorphic to the graph given in Figure 6(b), where $A=\left\{x_{1}, x_{2}, y_{1}, y_{2}, z_{1}, z_{2}, z_{3}, z_{4}, z_{5}\right\}$. But then $e_{G}(A, V(G) \backslash A)=6$, contrary to $e_{G}(V(G) \backslash A, A) \geqslant 8$ because $n=11$ and $d_{G}(v)=5$ for any $v \in V(G) \backslash A$.

This completes the proof of Theorem 4 . 


\section{Proof of Theorem 5}

To establish the desired lower bound, note that the planar graph $K_{2}+(n-2) K_{1}$ is $\left(K_{1}+2 K_{2}\right)$-free for all $n \geqslant 5$. Hence, $e x_{\mathcal{p}}\left(n, K_{1}+2 K_{2}\right) \geqslant 2 n-3$ for all $n \geqslant 5$. In particular, $e x_{\mathcal{p}}\left(5, K_{1}+2 K_{2}\right) \geqslant 7$. We next show that every $\left(K_{1}+2 K_{2}\right)$-free planar graph on $n \geqslant 5$ vertices has at most $19 n / 8-4$ edges. We proceed the proof by induction on $n$. Assume first $n=5$. Then $e x_{\mathcal{P}}\left(5, K_{1}+2 K_{2}\right)=7$, because the only plane triangulation on five vertices, given in Figure $4(\mathrm{a})$, is not $\left(K_{1}+2 K_{2}\right)$-free, and any $T_{5}^{-}$is not $\left(K_{1}+2 K_{2}\right)$ free. Hence, $e x_{\mathcal{p}}\left(n, K_{1}+2 K_{2}\right)=7<19 n / 8-4$ when $n=5$. So we may assume that $n \geqslant 6$. Let $G$ be a $\left(K_{1}+2 K_{2}\right)$-free plane graph on $n \geqslant 6$ vertices. Assume there exists a vertex $u \in V(G)$ with $d_{G}(u) \leqslant 2$. By the induction hypothesis, $e(G \backslash u) \leqslant 19(n-1) / 8-4$ and so $e(G)=e(G \backslash u)+d_{G}(u) \leqslant 19(n-1) / 8-4+2<19 n / 8-4$, as desired. So we may assume that $\delta(G) \geqslant 3$. Next, assume $G$ is disconnected. Then each component of $G$ either is isomorphic to $K_{4}$ or has at least six vertices because $\delta(G) \geqslant 3$. Let $G_{1}, \ldots, G_{p}, G_{p+1}, \ldots, G_{p+q}$ be all components of $G$ such that $\left|G_{1}\right|=\cdots=\left|G_{p}\right|=4$ and $6 \leqslant\left|G_{p+1}\right| \leqslant \cdots \leqslant\left|G_{p+q}\right|$, where $p \geqslant 0$ and $q \geqslant 0$ are integers with $p+q \geqslant 2$ and $\left|G_{p+1}\right|+\cdots+\left|G_{p+q}\right|=n-4 p$. Then $e\left(G_{i}\right)=6$ for all $i \in[p]$, and $e\left(G_{j}\right) \leqslant \frac{19\left|G_{j}\right|}{8}-4$ for all $j \in\{p+1, \ldots, p+q\}$ by the induction hypothesis. Therefore,

$$
\begin{aligned}
e(G) & \leqslant 6 p+\frac{19\left(\left|G_{p+1}\right|+\cdots+\left|G_{p+q}\right|\right)}{8}-4 q \\
& =\frac{19 n}{8}-\frac{7 p}{2}-4 q \\
& \leqslant \frac{19 n}{8}-\frac{7(p+q)}{2}<\frac{19 n}{8}-4,
\end{aligned}
$$

as desired. So we may further assume that $G$ is connected. Then $G$ has no faces of size at most two. Hence,

$$
2 e(G)=3 f_{3}+\sum_{i \geqslant 4} i f_{i} \geqslant 3 f_{3}+4\left(f-f_{3}\right)=4 f-f_{3},
$$

which implies that

$$
f \leqslant e(G) / 2+f_{3} / 4 \text {. }
$$

Note that each 3-vertex is incident with at most three distinct 3-faces in $G$. Furthermore, since $G$ is $\left(K_{1}+2 K_{2}\right)$-free, we see that for all $j \geqslant 4$, each $j$-vertex is incident with at most two distinct 3-faces in $G$. Let $U \subseteq V(G)$ denote the set of 3-vertices each incident with exactly three distinct 3-faces in $G$. Then $U$ must be an independent set in $G$ because $G$ is connected. Furthermore, no two vertices in $U$ have a common neighbor in $G$, because $G$ is $\left(K_{1}+2 K_{2}\right)$-free. Thus, $4|U| \leqslant n$ and so $|U| \leqslant n / 4$. It follows that

$$
3 f_{3} \leqslant 3|U|+2(n-|U|)=2 n+|U| \leqslant 9 n / 4
$$

which implies that $f_{3} \leqslant 3 n / 4$. This, together with $(1)$, further implies that $f \leqslant e(G) / 2+$ $3 n / 16$. By Euler's formula, $n-2=e(G)-f \geqslant e(G) / 2-3 n / 16$. Hence, $e(G) \leqslant 19 n / 8-4$, as desired. 


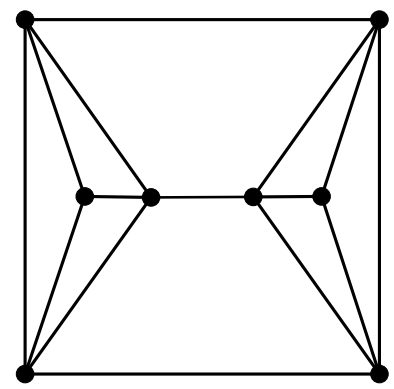

(a)

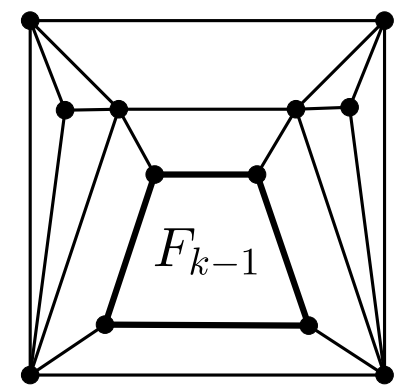

(b)

Figure 7: The construction of $F_{k}$.

From the proof above, we see that equality in $e(G) \leqslant 19 n / 8-4$ is achieved for $n$ if and only if equalities hold in both $(1)$ and $(2)$, and in $4|U| \leqslant n$. This implies that $e(G)=19 n / 8-4$ for $n$ if and only if $G$ is a connected, $\left(K_{1}+2 K_{2}\right)$-free plane graph on $n$ vertices satisfying: $\delta(G) \geqslant 3$; each 3 -vertex in $G$ is incident with exactly three distinct 3-faces; each vertex of degree at least 4 in $G$ is incident with exactly two distinct 3 -faces; each face is either a 3 -face or a 4 -face. We next construct such an extremal plane graph for $n$ and $K_{1}+2 K_{2}$. Let $n=8(k+1)$ for some integer $k \geqslant 0$. Let $F_{0}$ be the graph depicted in Figure $7(\mathrm{a})$, we then construct $F_{k}$ of order $n$ recursively for all $k \geqslant 1$ via the illustration given in Figure $7(\mathrm{~b})$ : the entire graph $F_{k-1}$ is placed into the center quadrangle of Figure 7(b) (in such a way that the center bold quadrangle of Figure 7(b) is identified with the outer quadrangle of $\left.F_{k-1}\right)$. One can check that $F_{k}$ is $\left(K_{1}+2 K_{2}\right)$-free with $n=8(k+1)$ vertices and $19 n / 8-4$ edges for all $k \geqslant 0$.

This completes the proof of Theorem 5 .

\section{$5 \quad$ Proof of Theorem 6}

By Proposition $3(\mathrm{~b}), e x_{\mathcal{P}}\left(n, K_{1, t}\right)=3 n-6$ for all $n \geqslant t+1 \geqslant 8$. So we may assume that $t \leqslant 6$. We next show that $e x_{\mathcal{P}}\left(n, K_{1,6}\right)=3 n-6$ for all $n \in\{7,8,9,10,12\}$. Let $J_{a}, J_{b}, J_{c}$ be the plane graphs given in Figure 8. Let $J_{a}^{\prime}$ be the plane triangulation obtained from $J_{a}$ by adding a new vertex adjacent to $x_{1}, x_{2}, x_{3}, J_{b}^{\prime}$ be the plane triangulation obtained from $J_{b}$ by first deleting the edge $x_{1} x_{3}$ and then adding a new vertex adjacent to $x_{1}, x_{2}, x_{3}, x_{4}$, and $J_{c}^{\prime}$ be the plane triangulation obtained from $J_{c}$ by first deleting the edge $x_{1} x_{3}$ and then adding one new vertex adjacent to $x_{1}, x_{2}, x_{3}, x_{4}, x_{5}$. Then the plane triangulations $J_{a}, J_{a}^{\prime}, J_{b}, J_{b}^{\prime}$ and $J_{c}^{\prime}$ are $K_{1,6}$-free because each of them has maximum degree 5. Hence, $e x_{\mathcal{P}}\left(n, K_{1,6}\right)=3 n-6$ for all $n \in\{7,8,9,10,12\}$. By Lemma 8, no plane triangulation on 11 vertices has maximum degree at most 5 . Hence, every plane triangulation on $n \in\{11,13,14\}$ vertices has maximum degree at least 6 . This implies that $e x_{\mathcal{p}}\left(n, K_{1,6}\right) \leqslant 3 n-7$ for all $n \in\{11,13,14\}$. Since $J_{c}$ given in Figure 8 is a $K_{1,6}$-free plane graph with $n=11$ vertices and $3 n-7$ edges, we have $e x_{\mathcal{p}}\left(n, K_{1,6}\right)=3 n-7$ when 


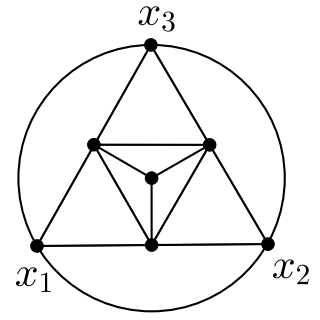

$J_{a}$

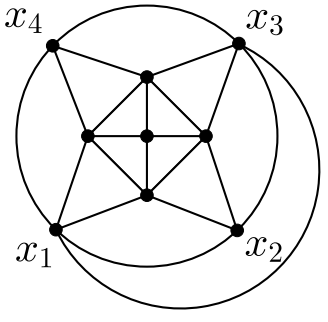

$J_{b}$

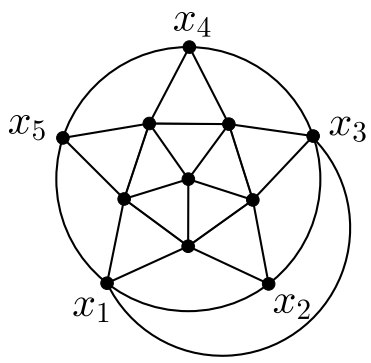

$J_{c}$

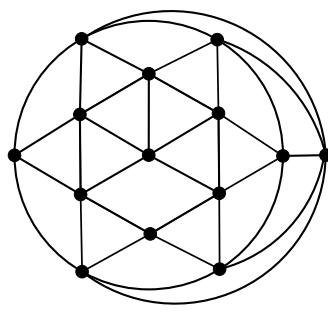

$J_{d}$

Figure 8: The graphs $J_{a}, J_{b}, J_{c}$ and $J_{d}$.

$n=11$. By Lemma 8, there does not exist any planar graphs on $n \in\{13,14\}$ vertices with $3 n-7$ edges and maximum degree at most 5 . It follows that $e x_{\mathcal{P}}\left(n, K_{1,6}\right) \leqslant 3 n-8$ when $n \in\{13,14\}$. Let $J_{c}^{\prime \prime}$ be the plane graph obtained from $J_{c}$ by first deleting the edge $x_{1} x_{3}$ and then adding two new adjacent vertices $y_{1}, y_{2}$ such that $y_{1}$ is adjacent to $x_{1}, x_{2}, x_{3}$ and $y_{2}$ is adjacent to $x_{4}, x_{5}$. Then $J_{c}^{\prime \prime}$ and the graph $J_{d}$ given in Figure 8 are $K_{1,6}$-free plane graph with $n \in\{13,14\}$ vertices and $3 n-8$ edges. Hence, $e x_{\mathcal{P}}\left(n, K_{1,6}\right)=3 n-8$ when $n \in\{13,14\}$.

It is easy to see that $e x_{p}\left(n, K_{1,3}\right)=n$ for all $n \geqslant 4$, because every $K_{1,3}$-free planar graph has maximum degree at most 2 and the planar graph $C_{n}$ is $K_{1,3}$-free with $n$ edges. We next show that $e x_{\mathcal{P}}\left(n, K_{1,4}\right)=\lfloor 3 n / 2\rfloor$ for all $n \geqslant 5$. Clearly, ex $\left(n, K_{1,4}\right) \leqslant\lfloor 3 n / 2\rfloor$ for all $n \geqslant 5$, because every $K_{1,4}$-free planar graph has maximum degree at most 3 . Next, for all $n \geqslant 5$, the planar graph obtained from $C_{n}$ by adding a matching of size $\lfloor n / 2\rfloor$ is $K_{1,4}$-free with $\lfloor 3 n / 2\rfloor$ edges. Hence, $e x_{\mathcal{P}}\left(n, K_{1,4}\right)=\lfloor 3 n / 2\rfloor$ for all $n \geqslant 5$. To determine $e x_{\mathcal{P}}\left(n, K_{1,5}\right)$ for all $n \geqslant 6$, since every $K_{1,5}$-free planar graph on $n \geqslant 6$ vertices has maximum degree at most 4 , we have $e x_{\mathcal{P}}\left(n, K_{1,5}\right) \leqslant 2 n$ for all $n \geqslant 6$. Let $J_{a}^{\prime \prime}$ be the plane triangulation obtained from $J_{a}$ by deleting the unique 3 -vertex. Since $J_{a}^{\prime \prime}$ is $K_{1,5}$-free plane graph on $n=6$ vertices with $2 n$ edges, we have $e x_{\mathcal{p}}\left(n, K_{1,5}\right)=2 n$ when $n=6$. By Lemma 8, no planar graph with $n=7$ vertices and $2 n$ edges has maximum degree at most 4. Hence, $e x_{\mathcal{P}}\left(n, K_{1,5}\right) \leqslant 2 n-1$ when $n=7$. Let $J_{a}^{\prime \prime \prime}$ be the plane graph obtained from $J_{a}^{\prime \prime}$ by first deleting the edge $x_{1} x_{2}$ and then adding a new vertex adjacent to $x_{1}, x_{2}$ only. Note that $J_{a}^{\prime \prime \prime}$ is a $K_{1,5}$-free plane graph on $n=7$ vertices with $2 n-1$ edges, we see that $e x_{\mathcal{p}}\left(n, K_{1,5}\right)=2 n-1$ when $n=7$. Next, for all $n \geqslant 8$, let $C$ be a cycle on $2\lfloor n / 2\rfloor$ vertices with vertices $u_{1}, \ldots, u_{\left\lfloor\frac{n}{2}\right\rfloor}, w_{\left\lfloor\frac{n}{2}\right\rfloor}, \ldots, w_{1}$ in order. Let $H$ be the plane graph obtained from $C$ by adding the path with vertices $w_{1}, u_{2}, w_{2}, u_{3}, \ldots, w_{\left\lfloor\frac{n}{2}\right\rfloor-1}, u_{\left\lfloor\frac{n}{2}\right\rfloor}$ in order. When $n$ is even, the planar graph $H+u_{1} u_{\left\lfloor\frac{n}{2}\right\rfloor}+u_{1} w_{\left\lfloor\frac{n}{2}\right\rfloor}+w_{1} w_{\left\lfloor\frac{n}{2}\right\rfloor}$ is $K_{1,5}$-free with $2 n$ edges. When $n$ is odd, let $H^{\prime}$ be obtained from $H$ by first deleting the edge $u_{2} u_{3}$ and then adding a new vertex $u$ adjacent to $u_{2}$ and $u_{3}$. Then the planar graph $H^{\prime}+u u_{1}+u u_{\left\lfloor\frac{n}{2}\right\rfloor}+w_{1} w_{\left\lfloor\frac{n}{2}\right\rfloor}+u_{1} w_{\left\lfloor\frac{n}{2}\right\rfloor}$ is $K_{1,5}$-free with $2 n$ edges. Hence, $e x_{\mathcal{P}}\left(n, K_{1,5}\right)=2 n$ for all $n \geqslant 8$.

It remains to show that $e x_{\mathcal{P}}\left(n, K_{1,6}\right)=\lfloor 5 n / 2\rfloor$ for all $n \geqslant 15$. Clearly, ex $x_{\mathcal{P}}\left(n, K_{1,6}\right) \leqslant$ $\lfloor 5 n / 2\rfloor$ for all $n \geqslant 15$, because every $K_{1,6}$-free planar graph on $n \geqslant 15$ vertices has maximum degree at most 5 . We next show that $e x_{\mathcal{P}}\left(n, K_{1,6}\right) \geqslant\lfloor 5 n / 2\rfloor$ for all $n \geqslant 15$. 
Let $n:=4 q+r \geqslant 15$, where $q \geqslant 3$ and $r \in\{0,1,2,3\}$. Let $p \in\{q, q+1\}$. Let $C^{1}$ and $C^{2}$ be two vertex-disjoint cycles with vertices $x_{1}, x_{2}, \ldots x_{q}$ in order and $y_{1}, y_{2}, \ldots y_{p}$ in order, respectively. Let $C^{3}$ be a cycle of length $q+p$ with vertices $b_{1}, a_{1}, b_{2}, a_{2}, \ldots b_{q}, a_{q}$ in order when $p=q$, and $b_{1}, a_{1}, b_{2}, a_{2}, \ldots b_{q}, a_{q}, b_{q+1}$ in order when $p=q+1$. Let $R_{p}$ be the plane graph on $2 q+2 p$ vertices obtained from disjoint copies of $C^{1}, C^{2}$ and $C^{3}$ by making $x_{i}$ adjacent to $\left\{a_{i}, b_{i}, b_{i+1}\right\}$, and $y_{j}$ adjacent to $\left\{b_{j}, a_{j-1}, a_{j}\right\}$ for all $1 \leqslant i \leqslant q$ and $1 \leqslant j \leqslant p$, where all arithmetic on the indices $i+1$ and $j-1$ here are done modulo

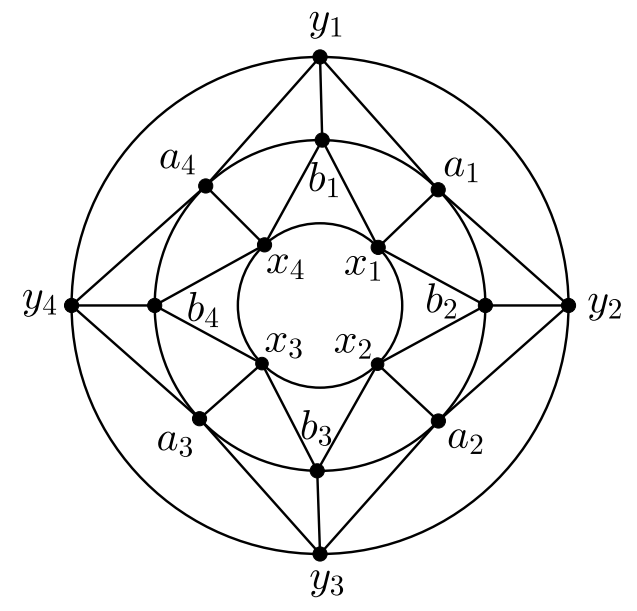

(a)

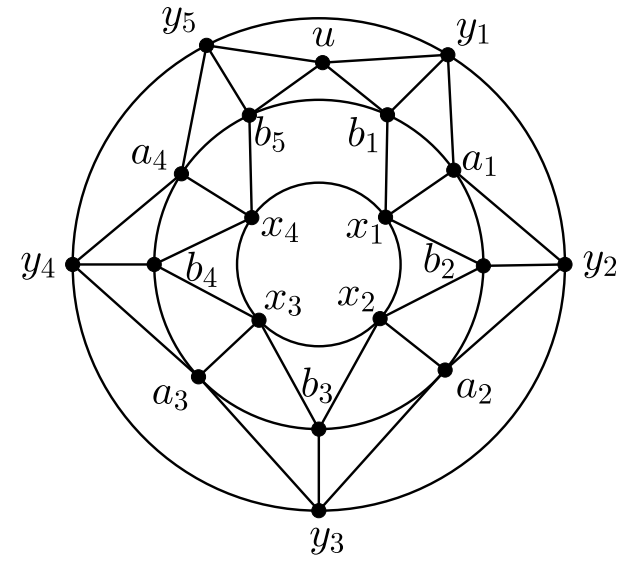

(b)

Figure 9: Almost 5-regular plane graphs on $4 q+r$ vertices when $q=4$ and $r \in\{0,3\}$.

$p$. Then $R_{p}$ is $K_{1,6}$-free planar graph with $10 q$ edges when $p=q$ and $10 q+3$ edges when $p=q+1$. The construction of $R_{p}$ on $4 q$ vertices when $q=4$ is depicted in Figure 9(a). When $n=4 q$ for $q \geqslant 4$, the planar graph $R_{p}$ with $p=q$ is $K_{1,6}$-free with $10 q$ edges and so $e x_{\mathcal{p}}\left(n, K_{1,6}\right)=10 q=5 n / 2$. When $n=4 q+1$ for $q \geqslant 4$, let $R^{1}$ be obtained from $R_{p}-y_{2} y_{3}-y_{1} y_{p}$ with $p=q$ by adding a new vertex $u$ adjacent to $y_{2}$ and $y_{3}$. Then the planar graph $R^{1}+u y_{1}+u y_{p}$ is $K_{1,6}$-free with $10 q+2$ edges. Hence, $e x_{\mathcal{p}}\left(n, K_{1,6}\right)=10 q+2=\lfloor 5 n / 2\rfloor$ when $n=4 q+1$ for $q \geqslant 4$. When $n=4 q+2$ for $q \geqslant 4$, let $R^{2}$ be obtained from $R_{p}-y_{2} y_{3}-y_{1} y_{p}-x_{2} x_{3}-x_{1} x_{p}-b_{1} a_{p}$ with $p=q$ by adding two new vertices $u$ adjacent to $y_{2}$ and $y_{3}$ and $v$ adjacent to $x_{2}$ and $x_{3}$. Then the planar graph $R^{2}+u y_{1}+u y_{p}+u a_{q}+v x_{1}+v x_{q}+v b_{1}$ is $K_{1,6}$-free with $10 q+5$ edges. Hence, $e x_{\mathcal{P}}\left(n, K_{1,6}\right)=10 q+5=5 n / 2$ when $n=4 q+2$ for $q \geqslant 4$. When $n=4 q+3$ for $n \geqslant 3$, the planar graph obtained from $R_{p}$ with $p=q+1$ by adding a new vertex $u$ adjacent to $y_{1}, b_{1}, y_{p}, b_{p}$, given in Figure 9 (b) when $q=4$, is $K_{1,6}$-free with $10 q+7$ edges. Hence, $e x_{\mathcal{p}}\left(n, K_{1,6}\right)=10 q+7=\lfloor 5 n / 2\rfloor$ when $n=4 q+3$ for $q \geqslant 3$.

This completes the proof of Theorem 6 . 


\section{Proof of Theorem 7}

Since the plane triangulations $J_{a}, J_{a}^{\prime}, J_{b}$ and $J_{b}^{\prime}$ constructed in the proof of Theorem 6 is $\left(K_{1}+3 K_{2}\right)$-free, we see that $e x_{\mathcal{p}}\left(n, K_{1}+3 K_{2}\right)=3 n-6$ for all $n \in\{7,8,9,10\}$. To determine $e x_{\mathcal{P}}\left(11, K_{1}+3 K_{2}\right)$, note that the plane graph $J_{c}$ given in Figure 8 with $n=11$ vertices and $3 n-7$ edges is $\left(K_{1}+3 K_{2}\right)$-free. Thus ex $\left(n, K_{1}+3 K_{2}\right) \geqslant 3 n-7$ when $n=11$. By Lemma 8, no plane triangulation on 11 vertices has maximum degree at most 5. Hence, every plane triangulation on 11 vertices must contain a vertex of degree at least 6 (and so contains a copy of $K_{1}+3 K_{2}$ ), which implies that $e x_{\mathcal{p}}\left(n, K_{1}+3 K_{2}\right)=3 n-7$ when $n=11$. Since every $K_{1,6}$-free graph is certainly $\left(K_{1}+3 K_{2}\right)$-free, by Theorem 6 , $e x_{\mathcal{P}}\left(n, K_{1}+3 K_{2}\right)=e x_{\mathcal{P}}\left(n, K_{1,6}\right)=3 n-6$ when $n=12, e x_{\mathcal{P}}\left(n, K_{1}+3 K_{2}\right) \geqslant e x_{\mathcal{P}}\left(n, K_{1,6}\right)=$ $3 n-8$ when $n \in\{13,14\}$, and $e x_{\mathcal{P}}\left(n, K_{1}+3 K_{2}\right) \geqslant e x_{\mathcal{P}}\left(n, K_{1,6}\right)=\lfloor 5 n / 2\rfloor$ when $n \geqslant 15$. Since every plane triangulation on $n \in\{13,14\}$ vertices has maximum degree at least 6 , we see that $e x_{\mathcal{P}}\left(n, K_{1}+3 K_{2}\right) \leqslant 3 n-7$ when $n \in\{13,14\}$. By Lemma 8 , every $T_{n}^{-}$with $n \in\{13,14\}$ has maximum degree at least 6 and so contains a copy of $K_{1}+3 K_{2}$. It follows that $e x_{\mathcal{P}}\left(n, K_{1}+3 K_{2}\right)=3 n-8<17 n / 6-4$ when $n \in\{13,14\}$.

We next show that every $\left(K_{1}+3 K_{2}\right)$-free planar graph $G$ on $n \geqslant 13$ vertices has at most $17 n / 6-4$ edges. We proceed the proof by induction on $n$. This is trivially true when $n \in\{13,14\}$. So we may assume that $n \geqslant 15$. Assume there exists a vertex $u \in V(G)$ with $d_{G}(u) \leqslant 2$. By the induction hypothesis, $e(G \backslash u) \leqslant 17(n-1) / 6-4$ and so $e(G)=e(G \backslash u)+d_{G}(u) \leqslant 17(n-1) / 6-4+2<17 n / 6-4$, as desired. So we may assume that $\delta(G) \geqslant 3$. Assume next that $G$ is disconnected. Let $G_{1}, \ldots, G_{p}, G_{p+1}, \ldots, G_{p+q}$ be all components of $G$ such that $\left|G_{1}\right| \leqslant \cdots \leqslant\left|G_{p}\right| \leqslant 12$ and $13 \leqslant\left|G_{p+1}\right| \leqslant \cdots \leqslant\left|G_{p+q}\right|$, where $p \geqslant 0$ and $q \geqslant 0$ are integers with $p+q \geqslant 2$ and $\left|G_{1}\right|+\cdots+\left|G_{p+q}\right|=n$. Then $e\left(G_{i}\right) \leqslant 3\left|G_{i}\right|-6$ for all $i \in[p]$, and $e\left(G_{j}\right) \leqslant 17\left|G_{j}\right| / 6-4$ for all $j \in\{p+1, \ldots, p+q\}$ by the induction hypothesis. Therefore,

$$
\begin{aligned}
e(G) & \leqslant 3\left(\left|G_{1}\right|+\cdots+\left|G_{p}\right|\right)-6 p+\frac{17\left(\left|G_{p+1}\right|+\cdots+\left|G_{p+q}\right|\right)}{6}-4 q \\
& =\frac{17 n}{6}-(6 p+4 q)+\frac{\left|G_{1}\right|+\cdots+\left|G_{p}\right|}{6} \\
& \leqslant \frac{17 n}{6}-(6 p+4 q)+2 p=\frac{17 n}{6}-4(p+q)<\frac{17 n}{6}-4,
\end{aligned}
$$

as desired. So we may further assume that $G$ is connected. Then $G$ has no faces of size at most two. Hence,

$$
2 e(G)=3 f_{3}+\sum_{i \geqslant 4} i f_{i} \geqslant 3 f_{3}+4\left(f-f_{3}\right)=4 f-f_{3},
$$

which implies that $f \leqslant e(G) / 2+f_{3} / 4$. Note that $n_{3}(G) \geqslant 0$ and $n_{5}(G)<n$; and for all $i \in\{3,4,5\}$, each $i$-vertex is incident with at most $i$-faces. Furthermore, for all $j \geqslant 6$, each $j$-vertex is incident with at most four 3 -faces because $G$ is $\left(K_{1}+3 K_{2}\right)$-free and $n \geqslant 15$. It follows that

$3 f_{3} \leqslant 3 n_{3}(G)+4 n_{4}(G)+5 n_{5}(G)+4\left(n-n_{3}(G)-n_{4}(G)-n_{5}(G)\right)=4 n-n_{3}(G)+n_{5}(G)<5 n$, 
which implies that $f_{3}<5 n / 3$. This, together with the fact that $f \leqslant e(G) / 2+f_{3} / 4$, further implies that $f<e(G) / 2+5 n / 12$. By Euler's formula, $n-2=e(G)-f>e(G) / 2-5 n / 12$. Hence, $e(G)<17 n / 6-4$.

This completes the proof of Theorem 7 .

\section{Concluding remarks}

The lower bound in Theorem 7 can be further improved when $n$ is divisible by 24 . To see this, let $n=24(k+1)$ for some integer $k \geqslant 0$. Let $R_{5}$ be the 5 -regular plane graph on twelve vertices given in Figure 10(a), and let $G_{0}$ be the plane graph obtained from two disjoint copies of $R_{5}$ by adding three independent edges between their outer faces, as depicted in Figure 10(b). We construct $G_{k}$ of order $n$ recursively for all $k \geqslant 1$ via the illustration given in Figure 10(c): the entire graph $G_{k-1}$ is placed into the center quadrangle of Figure 10(c) (in such a way that the center bold quadrangle of Figure 10(c) is identified with the outer quadrangle of $\left.G_{k-1}\right)$. One can check that $G_{k}$ is $\left(K_{1}+3 K_{2}\right)$-free with $n=24(k+1)$ vertices and $67 n / 24-4$ edges for all $k \geqslant 0$.

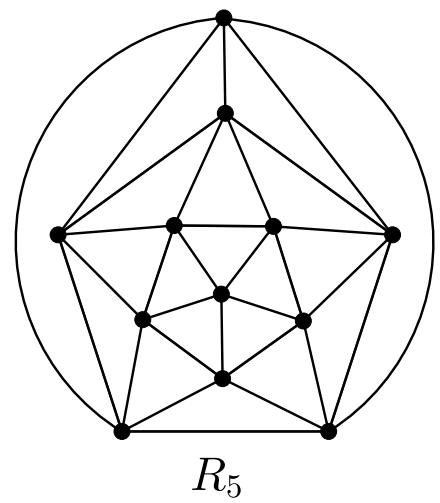

(a)

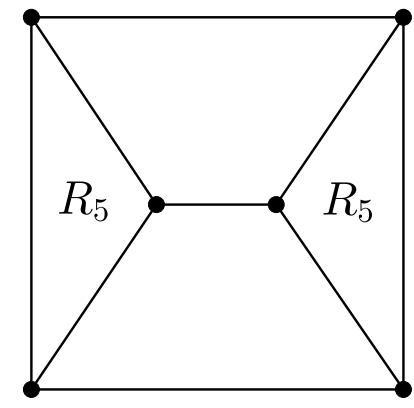

(b)

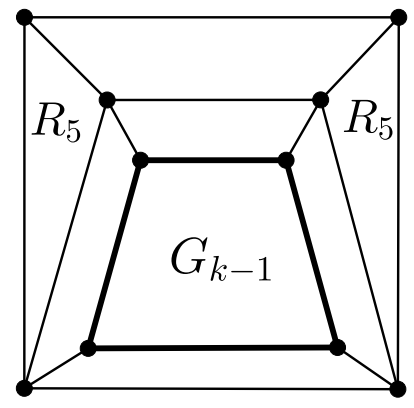

(c)

Figure 10: The construction of $G_{k}$.

As mentioned earlier, it seems non-trivial to determine $e x_{\mathcal{P}}(n, H)$ for all $K_{4}$-free planar graphs $H$ with exactly one vertex, say $u$, satisfying $d_{H}(u)=\Delta(H) \leqslant 6$ and $\Delta(H[N(u)]) \leqslant$ 2. We conclude this section by giving an upper bound (but not tight) for $e x_{\mathcal{p}}\left(n, K_{1}+H\right)$, where $H$ is a disjoint union of paths.

Theorem 9. Let $4 \leqslant t \leqslant 6$ be an integer and let $H$ be a graph on $t$ vertices such that $H$ is a disjoint union of paths. Then ex $_{\mathcal{P}}\left(n, K_{1}+H\right) \leqslant \frac{13(t-1) n}{4 t-2}-\frac{12(t-1)}{2 t-1}$ for all $n \geqslant t+1$.

Proof. Let $t$ and $H$ be given as in the statement. Since $H$ is a subgraph of $K_{1}+P_{t}$, it suffices to show that $e x_{\mathcal{P}}\left(n, K_{1}+P_{t}\right) \leqslant \frac{13(t-1) n}{4 t-2}-\frac{12(t-1)}{2 t-1}$ for all $n \geqslant t+1$. Let $G$ 
be a $\left(K_{1}+P_{t}\right)$-free planar graph on $n \geqslant t+1$ vertices. We next show that $e(G) \leqslant$ $\frac{13(t-1) n}{4 t-2}-\frac{12(t-1)}{2 t-1}$ by induction on $n$. This is trivially true when $n=t+1$ because $e(G) \leqslant$ $3(t+1)-6 \leqslant \frac{13(t-1)(t+1)}{4 t-2}-\frac{12(t-1)}{2 t-1}$ for $5 \leqslant t \leqslant 6$ and $e(G) \leqslant 3(t+1)-7 \leqslant \frac{13(t-1)(t+1)}{4 t-2}-\frac{12(t-1)}{2 t-1}$ for $t=4$. So we may assume that $n \geqslant t+2$. We may further assume that $\delta(G) \geqslant 3$ and $G$ is connected. Hence,

$$
2 e(G)=3 f_{3}+\sum_{i \geqslant 4} i f_{i} \geqslant 3 f_{3}+4\left(f-f_{3}\right)=4 f-f_{3},
$$

which implies that $f \leqslant e(G) / 2+f_{3} / 4$. Note that for all $3 \leqslant i \leqslant t-1$, each $i$-vertex is incident with at most $i$ many 3 -faces, and for all $j \geqslant t$, each $j$-vertex is incident with at most $(t-2)\left\lfloor\frac{j}{t-1}\right\rfloor$ many 3 -faces, because $G$ is $\left(K_{1}+P_{t}\right)$-free. It follows that

$$
\begin{aligned}
3 f_{3} & \leqslant \sum_{i=3}^{t-1} i \cdot n_{i}(G)+\sum_{j \geqslant t}(t-2)\left\lfloor\frac{j}{t-1}\right\rfloor \cdot n_{j}(G) \\
& \leqslant \sum_{i=3}^{t-1}\left(\frac{i \cdot n_{i}(G)}{t-1}+\frac{(t-2) i \cdot n_{i}(G)}{t-1}\right)+\frac{t-2}{t-1} \sum_{j \geqslant t} j \cdot n_{j}(G) \\
& =\sum_{i=3}^{t-1} \frac{i \cdot n_{i}(G)}{t-1}+\frac{t-2}{t-1} \sum_{\ell \geqslant 3} \ell \cdot n_{\ell}(G) \\
& =\sum_{i=3}^{t-1} \frac{i \cdot n_{i}(G)}{t-1}+\left(\frac{t-2}{t-1} \cdot 2 e(G)\right) \\
& =\sum_{i=3}^{t-1} n_{i}(G)-\sum_{i=3}^{t-1} \frac{(t-1-i) \cdot n_{i}(G)}{t-1}+\left(\frac{t-2}{t-1} \cdot 2 e(G)\right) \\
& <n+\frac{t-2}{t-1} \cdot 2 e(G),
\end{aligned}
$$

which implies that $f_{3}<\frac{n}{3}+\frac{2(t-2)}{3(t-1)} \cdot e(G)$. This, together with the fact that $f \leqslant e(G) / 2+$ $f_{3} / 4$, further implies that $f \leqslant \frac{e(G)}{2}+\frac{n}{12}+\frac{(t-2)}{6(t-1)} \cdot e(G)=\frac{(4 t-5)}{6(t-1)} \cdot e(G)+\frac{n}{12}$. By Euler's formula, $n-2=e(G)-f \geqslant \frac{(2 t-1)}{6(t-1)} \cdot e(G)-\frac{n}{12}$. Hence, $e(G) \leqslant \frac{13(t-1) n}{4 t-2}-\frac{12(t-1)}{2 t-1}$.

This completes the proof of Theorem 9 .

\section{Acknowledgments}

Zi-Xia Song would like to thank Yongtang Shi and the Chern Institute of Mathematics at Nankai University for hospitality and support during her visit in June 2018.

\section{References}

[1] B. Bollobás. Modern Graph Theory. Springer, 2013. 
[2] G. Chen, R. J. Gould, F. Pfender and B. Wei. Extremal graphs for intersecting cliques. J. Combin. Theory Ser. B, 89:159-171, 2003.

[3] C. Dowden. Extremal $C_{4}$-free $/ C_{5}$-free planar graphs. J. Graph Theory, 83:213-230, 2016.

[4] T. Dzido. A note on Turán numbers for even wheels. Graphs Combin., 29:1305-1309, 2013.

[5] T. Dzido and A. Jastrzębski. Turán numbers for odd wheels. Discrete Math., 341:1150-1154, 2018.

[6] P. Erdős, Z. Füredi, R. J. Gould and D. S. Gunderson. Extremal graphs for intersecting triangles. J. Combin. Theory Ser. B, 64:89-100, 1995.

[7] P. Erdős and A. Stone. On the structure of linear graphs. Bull. Amer. Math. Soc., 52:1087-1091, 1946.

[8] Z. Füredi, Turán type problems, "Surveys in Combinatorics", London Math. Soc. Lecture Note Ser. 166, pages 253-300. Cambridge Univ. Press, 1991.

[9] Z. Füredi and T. Jiang. Hypergraph Turán numbers of linear cycles. J. Combin. Theory Ser. A, 123:252-270, 2014.

[10] Z. Füredi, T. Jiang and R. Seiver. Exact solution of the hypergraph Turán problem for k-uniform linear paths. Combinatorica, 34:299-322, 2014.

[11] P. Keevash. Hypergraph Turán problems, "Surveys in Combinatorics". London Math. Soc. Lecture Note Ser. 392, pages 83-139. Cambridge Univ. Press, 2011.

[12] A. Kostochka, D. Mubayi and J. Verstraëte. Turán problems and shadows I: Paths and cycles. J. Combin. Theory Ser. A, 129:57-79, 2015.

[13] Y. Lan, Y. Shi and Z-X. Song. Extremal Theta-free planar graphs. arXiv: 1711.01614v2, 2017.

[14] E. F. Schmeichel and S. L. Hakimi. On planar graphical degree sequences. SIAM J. Appl. Math., 32:598-609, 1977.

[15] P. Turán. On an extremal problem in graph theory. Mat. Fiz. Lapok., 48:436-452, 1941. 